\title{
Frequency-response Analysis of Shear Vibration of Long Structures due to Surface Excitation
}

\author{
S. K. Chakraborty \\ Department of Applied Mathematics, Birla Institute of Technology, Mesra, Ranchi-835215, India
}

\author{
S. K. Sarkar \\ Indian Railway Institute of Mechanical and Electrical Engineering, Jamalpur-811214, India
}

\author{
S. P. Bhattacharya \\ Department of Architecture, Birla Institute of Technology, Mesra, Ranchi-835215, India
}

(Received 1 April 2006; revised 18 December 2006; accepted 27 April 2007)

The article presents a qualitative and quantitative analysis of the dynamic response of long structures, such as long and high-rise building. A two-dimensional linear model of a building is devised. In this model, the building is represented as a constrained anisotropic plate subjected to base excitation. Consequently, the displacement of the plate is governed by a $2 \mathrm{D}$ wave equation with corresponding boundary conditions. The dynamic response of the system, due to base excitation, is being analysed by expanding the displacements in terms of the corresponding eigenfunctions. Also, an analytical expression describing the building motion of translational type is presented. Further analysis is then conducted to determine the response to rotation at the base of the building. The investigation reveals the existence of wave passage effect during the dynamic response of the structure under the optimum conditions on frequencies (low or high) for proper analysis of the resulting deformation of the foundation.

\section{Nomenclature}

- amplitude of the incident wave (under the consid-

eration of behaviour of the foundation)

$a_{n}, a_{n}^{*} \quad-$ Fourier coefficients

$b \quad-$ thickness of the foundation

$c \quad-$ phase velocity of the incoming wave

D $\quad$ - storey drift

$f, g \quad$ - complex functions

$\bar{g} \quad-$ complex conjugate of $\mathrm{g}$

$H \quad-$ storey height

$k \quad-$ wave number

$k_{x, n}, k_{z, n}$ - wave numbers along $x$ - and $z$-directions

$L \quad-$ length of the building

$v_{\text {tex }}-$ displacement due to torsional excitation

$\varphi_{\text {tex }}-$ torsional excitation of the building

$n \quad-$ integer variable (varies from 1 to $\infty$ )

$N \quad-$ largest interger (n)

$Q_{1}, Q_{3}-$ shear moduli of rigidity

$T-$ time period of the wave

$v-\quad-$ transverse displacement of the beam

$v_{\text {rot }} \quad$ - rotational excitation

$v_{\text {tran }}-$ translational excitation

$X_{n}(x) \quad$ - eigenfunction in the $x$-direction

$Z_{n}(z) \quad$ - eigenfunction in the $z$-direction

$\rho-$ density of the medium

$\beta_{x}, \beta_{z} \quad$ - shear wave velocities along $x$ - and $z$-directions

$\delta-$ deflection of the foundation

$\eta \quad-$ dimensionless length of the building

$\lambda_{\text {in }}-$ wave length of the incident wave

$1 / R \quad-$ foundation's curvature

$\tau_{\max }-$ maximum shear stress in the foundation $\zeta-$ dimensionless parameter

$\omega-$ angular frequency

$\omega_{n, k} \quad-$ resonance frequencies

$\Phi_{0} \quad$ - rotational angle of foundation

\section{INTRODUCTION}

In reality, it is a tough task to assess the seismic resistance of high-rise buildings. Such an assessment may be feasible on a high confidence level, if the building can sustain all credible ground motions that can occur at its location. It may even be expected that it will survive with no more damage than a prescribed level. To analyse the vibration of such buildings, dynamic models are considered to assume uniform translation of the foundation only. For excitation of short wave lengths, relative to the foundation dimensions, the model used in the response analyses should include the rotational effect of such wave excitations also. The inclusion depends on the property of structure and on the nature of its contact with the ground. The contact can be near the surface area, or over an extended surface, as this in true for most buildings. ${ }^{1}$ Tzenov and Boncheva ${ }^{2}$ and Tzenov ${ }^{3}$ noted the need for the two dimensional models of 'long-in-plan' buildings to account for the phase difference between the excitation at different points of the foundation. However, they did not consider the excitation to be propagating waves. The nature of the seismic energy transfer from the ground into the foundation of a building, as well as the transport of energy within the building itself, has been discussed by Krivelev. ${ }^{4}$ The authors discuss modelling of buildings and suggest methods for calculating the equivalent $P$ and $S$ wave velocities of the equivalent continuous model. Hanks and McGuire ${ }^{5}$ studied the char- 